\title{
Effects of (In)Accurate Empathy and Situational Valence on Attitudes towards Robots
}

\author{
Henriette Cramer*†, Jorrit Goddijn*, Bob Wielinga*, Vanessa Evers* \\ *Human-Computer Studies lab, University of Amsterdam, The Netherlands \\ Email: jorritgoddijn@gmail.com, \{wielinga,evers\}@ science.uva.nl \\ ${ }^{\dagger}$ SICS \& Mobile Life Centre, Kista, Sweden \\ Email: henriette@mobilelifecentre.org
}

\begin{abstract}
Empathy has great potential in human-robot interaction. However, the challenging nature of assessing the user's emotional state points to the importance of also understanding the effects of empathic behaviours incongruent with users' affective experience. A 3x2 between-subject video-based survey experiment $(\mathrm{N}=133)$ was conducted with empathic robot behaviour (empathically accurate, neutral, inaccurate) and valence of the situation (positive, negative) as dimensions. Trust decreased when empathic responses were incongruent with the affective state of the user. However, in the negative valence condition, reported perceived empathic abilities were greater when the robot responded as if the situation were positive.

Index Terms-human-robot interaction, social robots, empathy, emotional valence
\end{abstract}

\section{INTRODUCTION AND BACKGROUND}

Empathy can have profound positive effects on users' attitudes towards social robots [?], [?], [?], [?]. Responding to the user's (affective) experience in a socially appropriate manner, is considered key in achieving user trust and satisfaction, as well as compliance to requests [?], [?].

Bickmore [?] found that accurate empathic system reactions are more important in comforting the user than sophisticated, detailed affect recognition. However, reacting in an empathic manner does require recognising the user's emotional state. This is challenging as it requires an appraisal of a deeply personal, individual experience; mistakes are likely. This reinforces the importance of understanding how people respond to empathic capabilities if a robot behaves in a manner that is incongruent with the user's emotional experience.

Inaccurate emotional responses can indeed result in negative consequences on user ratings of an agent [?]. Virtual agents showing emotions incongruent to the situation are also less appreciated by users than those expressing no emotion at all [?]. Research, however, yet has to fully uncover the effects of empathic behaviour (including possible inaccurate responses) in different situations. Empathy for example could be more vital in negative than in positive contexts [?]. In this study, we sought to clarify the effects of (in)accurate emphatic robot behaviours on people's responses in different contexts by investigating participants' attitudes toward a robot team mate.

\section{STUDY}

Our video-based, online survey experiment investigated participant' attitudes toward a four-minute interaction (Fig.??) of a male user and a robot, playing an online collaborative game together (for a discussion of video-based methods, see e.g. [?]). The $2 \times 3$, between-subject experiment varied situational valence (negative vs. positive) and empathic accuracy (an empathically accurate, neutral agent and an empathically inaccurate agent), resulting in six randomly assigned conditions. The robot used in the resulting six videos was the Philips iCat, with a synthetic female voice. Data of 133 participants were analysed (mean age $30.5,53 \%$ male).

Situational valence was manipulated by having the team do well on the collaborative game and win, or not do well together and lose. Empathic accuracy was varied using the robot's verbal responses and facial expressions. The verbal and facial expressions in the empathically accurate condition were congruent to the situation (e.g. acknowledging a negative experience when losing), In the neutral condition the social robot made no statements about the person's affective state.

Participants' (negative) attitude towards robots in general was measured using the NARS scale [?].

Likert-type and semantic differential scales were used to measure our dependent variables, including perceived empathic ability, trust (dependability, credibility) and closeness.

\section{RESULTS}

Two-way ANOVAs with empathic accuracy and situational valence as factors were used to check for interaction effects. One-way ANOVAs with planned contrasts were used to test for main effects. For non-parametric data Kruskal-Wallis tests were used, followed up by Mann-Whitney tests.

\section{A. Interaction effects empathic accuracy $X$ valence}

A significant interaction effect was found between empathic accuracy and emotional valence of the situation for the perceived empathic ability of the robot $(\mathrm{F}(2,112)=4.326, \mathrm{p}=.015)$. Simple effects analysis showed a significant difference for empathy shown in a positive or negative context. Participants rated the empathically accurate robot as having greater empathic ability in the positive condition compared with the empathically inaccurate robot, but this effect of accuracy was not significant $(\mathrm{F}=1.18, \mathrm{p}=.31)$. However, when the team was losing (negative context), participants found the empathically inaccurate robot to have better empathic abilities compared with the empathically accurate robot $(\mathrm{F}=3.24, \mathrm{p}=.043)$. 


\section{B. Effects of accuracy of empathic robot behaviour}

A main effect was found for empathic accuracy of the robot on dependability $(\mathrm{F}(2,106)=13.498, \mathrm{p}<.001)$. Planned contrasts showed that the neutral and accurate empathic robot were perceived as more dependable than the empathic inaccurate robot. The accurately empathic robot however was not perceived as significantly more dependable than the neutral robot. Empathic accuracy of the robot also had a direct effect that bordered on significance $(\mathrm{p}=.050)$ on perceived source credibility $(F(2,106)=3.076)$. Post-hoc tests showed that while the empathically accurate robot scores for credibility appeared higher than for the inaccurately empathic robot, this difference was not significant (post-hoc LSD, $\mathrm{p}=.066$ ). The neutral robot however was found more credible (post-hoc LSD, $\mathrm{p}=.023$ ) than the inaccurate robot. Furthermore, empathic accuracy had a direct effect on perceived closeness between the human and the robot $(\mathrm{H}(2)=12.046, \mathrm{p}=.002)$. The relation between the actor and the robot was perceived as less close for the inaccurate robot compared with the neutral robot $(\mathrm{U}=716.500, \mathrm{p}=.015)$. The relationship was perceived as closest in the accurate condition, also significantly higher than in the inaccurate condition ( $\mathrm{U}=695.000, \mathrm{p}=.001$ ) However, no significant difference was found between scores on perceived closeness of the accurately empathic robot and the neutral robot $(\mathrm{U}=675.000, \mathrm{p}=.398)$.

\section{Effect situational emotional valence}

Emotional valence of the situation was found to have a direct effect on perceived dependability $(F(1,106)=8.114$, $\mathrm{p}=.005)$, credibility $(\mathrm{F}(1,106)=6.532, \mathrm{p}=.012)$ and perceived closeness $(\mathrm{H}(1)=4.047, \mathrm{p}=.044)$. Participants in the positive condition found the robot significantly more dependable, more credible, and rated the relationship of the robot and user as closer than participants in the negative condition.

\section{Effects of attitudes towards robots in general}

Participants with a more negative attitude towards robots perceived the robot as more machine-like $(\mathrm{F}(1,95)=8.526$, $\mathrm{p}=.004)$ and less human-like $(\mathrm{F}(1,95)=18.690, \mathrm{p}<.001)$. They also thought the robot had less empathic abilities $(\mathrm{F}(1,95)=8.374, \mathrm{p}=.005)$, assessed the robot as less dependable $(\mathrm{F}(1,95)=9.620, \mathrm{p}=.003)$ and as less credible $(\mathrm{F}(1,95)=7.735$, $\mathrm{p}=.007)$ and the human-robot relationship as less close $(\mathrm{F}(1,95)=11.315, \mathrm{p}=.001)$. Attitudes towards robots did not interact with effects of empathic accuracy or situational valence.

\section{Discussion ANd CONCLUSION}

Our findings confirm that inaccurate empathic behaviour can be detrimental to user attitudes toward a social robot. A interesting result of this study was that in a negative (losing the game) context, participants found the robot that responded inaccurately to have more empathic abilities; they appeared to prefer positive, instead of accurate, statements. This, while these inaccurate reactions did lower trust. These incongruent results on perceived empathic ability and attitudes show the importance of not only assessing whether attempts at social

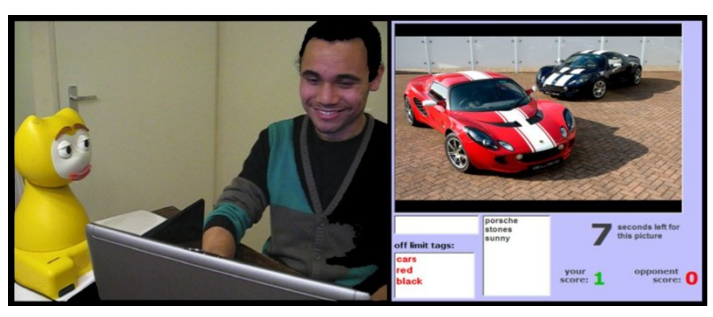

Fig. 1. Composite video (positive condition). Human-robot team left, game right (similar to the Google Image Labeller game). Presented simultaneously in split-screen manner, here adapted for clarity and printing.

behaviour are recognised, but also which effects behaviours have on user trust. They also illustrate the influence of how social ability is conceptualised. Appropriate social responses are highly context dependent and we need to consider for example that overall, in the negative condition, participants found the robot less credible and less dependable.

These results indicate the importance of showing restraint when implementing social behaviours. A robot's mistakes in assessing the affective state of the user and inaccurate empathic behaviour may have a profoundly detrimental effect on trust. So much so that, in certain contexts, robot designers may need to reconsider the introduction of empathic behaviours when the likelihood of inappropriate inferences on user affect is high.

\section{ACKNOWLEDGMENT}

We thank Karoenja Woudenberg, our video actor. This study was partially funded by the ICIS project, BSIK03024, by the Dutch Ministry of Economical Affairs under contract to the HCS lab of the University of Amsterdam.

\section{REFERENCES}

[1] J. Klein, Y. Moon, and R. W. Picard, "This computer responds to user frustration: Theory, design, and results," Interacting with Computers, vol. 14, no. 2, pp. 119-140, 2002.

[2] S. Brave, C. Nass, and K. Hutchinson, "Computers that care: investigating the effects of orientation of emotion exhibited by an embodied computer agent," Int. J. of Human-Computer Studies, vol. 62, no. 2, pp. 161-178, 2005

[3] K. Hone, "Empathic agents to reduce user frustration: The effects of varying agent characteristics," Interacting with Computers, vol. 18, no. 2, pp. 227-245, 2006.

[4] R. Picard and K. Liu, "Relative subjective count and assessment of interruptive technologies applied to mobile monitoring of stress." Int. J. of Human-Computer Studies, vol. 65, no. 4, pp. 361-375, 2007.

[5] T. Bickmore and D. Schulman, "Practical approaches to comforting users with relational agents," in Proceedings CHI'07, San Jose, CA, 2007.

[6] M. Ochs, C. Pelanchaud, and D. Sadek, "An empathic virtual dialog social robot to improve human-machine interaction," in Proceedings AAMAS'08, Estoril, Portugal, 2008, pp. 89-96.

[7] C. Becker, I. Wachsmuth, H. Prendinger, and M. Ishizuka, "Evaluating affective feedback of the $3 \mathrm{~d}$ agent max in a competitive cards game," in Proceedings ACII'05. Beijing, China: Springer, 2005.

[8] M. Killen and J. Smetana, Handbook of Moral Development, p. 528 New York, USA: Lawrence Erlbaum, 2006.

[9] S. Woods, M. Walters, K. Koay, and K. Dautenhahn, "Methodological issues in HRI: A comparison of live and video-based methods in robot to human approach direction trials." in Proceedings RO-MAN'06, Hatfield, UK, 2006, pp. 51-58.

[10] T. Nomura, T. Kanda, T. Suzuki, and K. Kato, "Psychology in human-robot communication," in Proceedings $\mathrm{RO}-M A N$ '04, Kurashiki, Okayama, Japan, 2004, pp. 35-40. 